\title{
Antixenosis Mechanism of Resistance to Helicoverpa armigera (Hub.) in Chickpea (Cicer arietinum Linn.)
}

\author{
E. Sree Latha* and H.C. Sharma
}

International Crops Research Institute for the Semi-Arid Tropics, Patancheru, 502 324, India

*Corresponding author

\section{A B S T R A C T}

Keywords

Helicoverpa armigera (Hub.),

Chickpea (Cicer

arietinum Linn.)

Article Info

Accepted:

10 January 2018

Available Online:

10 February 2018
The oviposition preference of $H$. armigera moths towards different genotypes of chickpea was studied under no choice, dual-choice and multichoice conditions in the laboratory at ICRISAT. Desi type genotypes (ICC 12475, ICC 12476, ICC 12477, ICC 12478, ICC 12479, ICC 12490 and ICC 14876) were not preferred for oviposition compared to kabuli type genotypes (ICC 12491, ICC 12493, ICC 12494, ICC 12495, ICC 12968, ICC 4973 and ICC 4962). The lines showing high and stable resistance to $H$. armigera can be used in chickpea improvement programs. The resistance mechanisms involved in these genotypes can be exploited to develop varieties resistant to $H$. armigera in chickpea.

\section{Introduction}

Host plant resistance is simple, convenient, and cheap and usually works well in combination with other forms of pest management, although it can have severe implications for the efficacy of some alternative pest management strategies such as bio-pesticides. In some cases, serious incompatibility does occur between natural plant resistance and other pest management approaches, so there is a great need to understand fully the mechanisms involved in resistance to ensure that antagonistic effects can be avoided (Stevenson et al., 2002).
During the course of evolution, plants acquire several defense mechanisms against insect pests to reduce the damage. The major mechanisms are antixenosis (non-preference), antibiosis, tolerance and escape potential (Painter, 1951). More of antibiosis, than antixenosis or tolerance has been reported in legume crops (Clement et al., 1992 and 1994). The studies made on host plant resistance will help to identify the genotypes with different types of mechanism of resistance and different sources of resistance can be combined to increase the diversity of resistant germplasm. Therefore the current studies were carried out to know the oviposition non preference of $H$. 
armigera on 10 chickpea genotypes belongs to desi and 8 genotypes to kabuli group.

\section{Materials and Methods}

Insect Culture: Larvae and adults of $H$. armigera used in feeding tests in the laboratory were obtained from a laboratory culture maintained at ICRISAT, Patancheru, India. The culture was established from, and regularly supplemented with field-collected larvae. Larvae were reared on a chickpea based diet (Armes et al., 1993) at $27^{\circ} \mathrm{C}$. Adults were kept at $25^{\circ} \mathrm{C}$ in a cage and mappyliners were provided as a substrate for oviposition. The moths were provided $10 \%$ honey solution on absorbent cotton for oviposition.

The oviposition preference of $H$. armigera moths towards different genotypes of chickpea was studied under no choice, dual-choice and multi-choice conditions in the laboratory at $25+2^{0} \mathrm{C}$ temperature and 65 to $90 \% \mathrm{RH}$. For oviposition tests, fresh flowering branches (20 $\mathrm{cm})$ brought from the field, were placed in a conical flask $(150 \mathrm{ml})$ filled with water and plugged with cotton wool. Three branches from a genotype (one straight and the other two in opposite directions) were placed in each conical flask.

For no-choice tests, a conical flask with chickpea branches of a genotype was placed at the center of cage. For dual choice tests, two flasks one with branch of a test genotype and the other with branches from a susceptible check (ICC 4918) were placed in a wooden cage $30 \times 30 \times 30 \mathrm{~cm}$. Three sides of the cage were fitted with glass, while the fourth one was covered with muslin cloth for aeration and facilitate release of moths inside the cage. A cup containing cotton wool soaked with sucrose solution (10\%) was placed in the center of each cage as a feed for adults. The chickpea plant branches offered as oviposition site were replaced every alternate day.
Five pairs of moths were released inside each cage. The eggs laid on chickpea branches were counted, removed gently with the help of camel hairbrush, and placed in a petri dish. The oviposition studies were conducted till the females seized to lay eggs.

Nonpreference for oviposition under multichoice conditions was studied by keeping all the 18 test genotypes (ICC 12475 (resistant check), ICC 12476, ICC 12477, ICC 12478, ICC 12479, ICC 12490, ICC 14876, ICC 4918 (susceptible check), ICC 12426, ICC 3137, ICC 12491, ICC 12492, ICC 12493, ICC 12494, ICC 12495, ICC 12968, ICC 4973 and ICC 4962) inside a wooden cage $(80 \times 70 \times 60$ $\mathrm{cm})$.

Conical flasks containing chickpea branches were arranged inside the wooden cage in completely randomized block design. Thirty pairs of adults were released inside the cage. Moths were provided with sucrose solution in a cotton swab.

Throughout the experiment, the moths were allowed to oviposit on the test genotypes for three consecutive nights. To avoid predation by the ants, tangle foot ${ }^{\mathrm{R}}$ glue was applied to all the four legs of the wooden table. Experiment was replicated three times.

Relative ovipositional preference $=$ (No. of eggs laid on standard variety $\mathrm{x}$ No. of eggs laid on test variety / No of eggs laid on Test variety + No. of eggs laid on standard variety) $\mathrm{x} 100$

Number of eggs laid were transformed to square root values $(\sqrt{0} .5+\mathrm{x})$, and the data were subjected to ANOVA under no-choice and multi-choice conditions. Two tailed student " $\mathrm{t}$ " test was performed on the mean number of eggs laid on the test genotypes to test the null hypothesis under dual-choice conditions. 


\section{Results and Discussion}

Under no choice conditions, lowest number of eggs were laid on resistant check, ICC 12475 (543), followed by ICC 12476 (793), ICC 12477 (818), and ICC 12479 (867). Highest number of eggs were recorded on ICC 4973 (1569), which were approximately three times greater than the eggs laid on resistant check, ICC 12475. Under multi-choice conditions, lowest number of eggs were laid on resistant check, ICC 12475 (423), followed by ICC 12476 (632), ICC 12477 (828), ICC 12426 (854) and ICC 12479 (878). Highest number of eggs was recorded on ICC 4962 (1686).

Under dual-choice conditions significantly lower number of eggs were laid on ICC 12475 and ICC 12476 compared to the susceptible check, ICC 4918 (Table 1). ICC 12475, ICC 12476, ICC 12477, ICC 12478, ICC 12479, ICC 12490 and ICC 14876 were not preferred for oviposition compared to ICC 4918 (susceptible check) under no-choice, dualchoice and multi-choice conditions. ICC 12491 was less preferred under no-choice and multi-choice conditions and ICC 12492 under dual-choice conditions. ICC 12426, ICC 3137, ICC 12493, ICC 12494, ICC 12495, ICC 12968, ICC 4973 and ICC 4962 were preferred for oviposition as compared to the susceptible check ICC 4918 (Tables 1 and 2).

More number of eggs was recorded on ICC 12426, ICC 3137, ICC 12491, ICC 12493, ICC 12494, ICC 12495, ICC 12968, ICC 4973 and ICC 4962 as compared to the susceptible check ICC 4918, under dual-choice condition but the differences were not significant (Table 2).

Oviposition in $H$. armigera usually starts some hours after dusk initially alternating with feeding, later becoming the predominant activity until soon after midnight (Pearson and Darling, 1958). Moths are highly selective in their choice of host plant in a suitable condition of development (Hardwick, 1965). On chickpea the eggs are laid mostly on leaves on underside when the plants are still very small. In contrast to other hosts, oviposition on chickpea declines from the onset of flowering (King, 1994).

The physiological state of an insect is a product of numerous interacting variations like age, feeding status and egg load etc. Egg load is one of several factors that may affect host selection behavior (Singer 1982; Fitt, 1986; Blaney and Simmonds, 1990 and Courtney and Kibota, 1990). Females with higher egg load may be less discriminating and more accepting of low ranking host plant (Minkenberg et al., 1992 and Prokopy et al., 1994). Mustapha et al., (1998) reported that female moths were less discriminating against cowpea (a low ranked host) relative to maize (a high ranked host) when egg load increased. Sison et al., (1993) conducted studies on the ovipositional preference of $H$. armigera among short duration pigeon pea genotypes and reported that flower colour influences the choice for oviposition. Sison et al., (1996) reported antixenosis as one of the mechanisms of resistance to $H$. armigera in chickpea.

Srivastava and Srivastava (1989) reported oviposition non-preference as the cause of observed differences in pod damage among eight chickpea genotypes. They found direct relationship between the number of eggs laid and larval abundance. This clearly shows that ovipositional non-preference was mainly responsible for resistance expressed by the host genotypes, rather than larval preference and antibiosis. These results agree with results of Lateef (1985). Cowgill and Lateef (1996) screened seven genotypes in the field for ovipositional non-preference to $H$. armigera. Fewer eggs were recorded on ICC 506, than the susceptibility controls. These observations were confirmed by the laboratory studies. 
Table.1 Oviposition preference of $H$. armigera among chickpea genotypes in single choice and multi choice cage tests under laboratory conditions

\begin{tabular}{|c|c|c|c|c|c|c|c|c|}
\hline \multirow[t]{2}{*}{ Genotype } & \multicolumn{4}{|c|}{ Single choice } & \multicolumn{4}{|c|}{ Multi choice } \\
\hline & $\begin{array}{c}\text { Mean No. of } \\
\text { eggs }\end{array}$ & $\sqrt{-1} x+0.5$ & $\pm \mathrm{SE}$ (3 rep) & ROP* & $\begin{array}{c}\text { Mean No. } \\
\text { of eggs }\end{array}$ & $\sqrt{-x}+0.5$ & $\pm \mathrm{SE}$ (3 rep) & $\mathrm{ROP} *$ \\
\hline ICC 12476 & 793.5 & (23.475 & $\pm 0.9584)$ & -21.5 & 632 & (22.947 & $\pm 0.865)$ & -25.0 \\
\hline ICC 12477 & 818.0 & (23.789 & $\pm 1.2505)$ & -20.0 & 828 & $(25.210$ & $\pm 0.274)$ & -12.0 \\
\hline ICC12478 & 992.0 & $(25.555$ & $\pm 1.8489)$ & -10.6 & 939 & $(26.053$ & $\pm 0.015)$ & -5.7 \\
\hline ICC 12479 & 867.0 & (24.318 & $\pm 1.4495)$ & -17.2 & 878 & (25.686 & $\pm 0.328)$ & -9.1 \\
\hline ICC 12490 & 921.0 & (24.348 & $\pm 2.0329)$ & -14.3 & 692 & $(23.753$ & $\pm 1.687)$ & -20.7 \\
\hline ICC 14876 & 916.5 & $(25.579$ & $\pm 0.4593)$ & -14.5 & 899 & (25.942 & $\pm 0.347)$ & -7.9 \\
\hline ICC 12426 & 1412.5 & (31.997 & $\pm 0.4062)$ & 7.0 & 854 & (28.108 & $\pm 3.220)$ & -10.4 \\
\hline ICC 3137 & 1369.5 & (31.184 & $\pm 1.0020)$ & 5.5 & 1189 & (34.439 & $\pm 1.692)$ & 6.1 \\
\hline ICC 12491 & 1143.0 & (28.443 & $\pm 1.2217)$ & -3.6 & 909 & $(30.133$ & $\pm 0.657)$ & -7.3 \\
\hline ICC 12492 & 1438.5 & (31.049 & $\pm 1.8438)$ & 7.9 & 1390 & (33.903 & $\pm 0.037)$ & 13.8 \\
\hline ICC 12493 & 1363.0 & (31.044 & $\pm 0.8455)$ & 5.2 & 1496 & (33.709 & $\pm 1.223)$ & 17.4 \\
\hline ICC 12494 & 1404.5 & (31.146 & $\pm 1.3805)$ & 6.7 & 1256 & (32.290 & $\pm 0.637)$ & 8.8 \\
\hline ICC 12495 & 1392.5 & $(30.270$ & $\pm 1.6352)$ & 6.3 & 1378 & (31.847 & $\pm 0.557)$ & 13.4 \\
\hline ICC 12968 & 1290.5 & (29.943 & $\pm 0.9434)$ & 2.5 & 1176 & (29.854 & $\pm 0.410)$ & 5.5 \\
\hline ICC 4973 & 1569.5 & (33.631 & $\pm 0.6615)$ & 12.2 & 1572 & (35.086 & $\pm 0.434)$ & 19.8 \\
\hline ICC 4962 & 1477.5 & (32.962 & $\pm 0.0075)$ & 9.2 & 1686 & (35.906 & $\pm 1.940)$ & 23.1 \\
\hline \multicolumn{9}{|l|}{ Checks } \\
\hline ICC $12475(\mathrm{R})$ & 543.5 & (20.137 & $\pm 0.0124)$ & -38.6 & 423 & (18.680 & $\pm 0.867)$ & -42.7 \\
\hline ICC 4918 (S) & 1227.5 & (29.989 & $\pm 0.3751)$ & 0.0 & 1053 & (29.586 & $\pm 0.599)$ & 0.0 \\
\hline
\end{tabular}

R-Resistant check, S-Susceptible check.

ROP*- Relative oviposition preference with respect to ICC 4918

Table.2 Relative oviposition preference of $H$. armigera towards chickpea genotypes under dual choice caged conditions

\begin{tabular}{|c|c|c|c|c|}
\hline \multirow[t]{2}{*}{ Genotype } & \multicolumn{2}{|c|}{ No. of eggs } & \multirow[t]{2}{*}{$\mathrm{t}$ (value) } & \multirow[t]{2}{*}{$\mathrm{ROP} *$} \\
\hline & Test genotype & ICC 4918 & & \\
\hline ICC 12476 & 103.0 & 174.3 & $1.81 *$ & -25.7 \\
\hline ICC 12477 & 82.5 & 129.8 & 1.18 & -22.3 \\
\hline ICC 12478 & 49.0 & 119.5 & 1.57 & -41.8 \\
\hline ICC 12479 & 75.2 & 137.6 & 1.19 & -29.3 \\
\hline ICC 12490 & 84.9 & 107.0 & 0.63 & -11.5 \\
\hline ICC 14876 & 81.0 & 148.4 & 1.44 & -29.3 \\
\hline ICC 12426 & 154.3 & 124.2 & -0.82 & 10.8 \\
\hline ICC 3137 & 142.8 & 102.5 & -1.00 & 16.4 \\
\hline ICC 12491 & 144.8 & 111.6 & -0.86 & 12.9 \\
\hline ICC 12492 & 114.2 & 127.3 & 0.37 & -5.4 \\
\hline ICC 12493 & 127.7 & 105.1 & -0.79 & 9.7 \\
\hline ICC 12494 & 126.4 & 104.8 & -0.73 & 9.3 \\
\hline ICC 12495 & 119.7 & 116.7 & -0.10 & 1.3 \\
\hline ICC 12968 & 134.3 & 109.3 & -0.71 & 10.3 \\
\hline ICC 4973 & 183.8 & 163.5 & -0.54 & 5.8 \\
\hline ICC 4962 & 148.2 & 134.7 & -0.44 & 4.8 \\
\hline ICC $12475(\mathrm{R})$ & 74.5 & 175.2 & $2.82 *$ & -40.3 \\
\hline
\end{tabular}

* Significant at 5\% probability, R- Resistant check;

Replications $=3 ;$ ROP $*$ Relaive oviposition preference with respect to ICC 4918 . 
Current studies ovipositional non-preference was not evident in long duration genotypes of chickpea (ICC 3137, ICC 12495, ICC 4973 and ICC 4962). Cowgill and Lateef (1996) reported non-significant oviposition in long duration chickpea genotypes. ICC 12475, ICC 12476, ICC 12477, ICC 12478, ICC 12479, ICC 12490 and ICC 14876 were not preferred for oviposition as compared to ICC 4918. Cowgill and Lateef (1996) reported that ovipositional non-preference is a component of resistance in ICC 12475.

Desi type genotypes (ICC 12475, ICC 12476, ICC 12477, ICC 12478, ICC 12479, ICC 12490 and ICC 14876) were not preferred for oviposition compared to kabuli type genotypes (ICC 12491, ICC 12493, ICC 12494, ICC 12495, ICC 12968, ICC 4973 and ICC 4962).

To collect the data on eggs per plant during the flowering and poding stage it is difficult to record in field. It becomes difficult to obtain reliable data on genotypic resistance /susceptibility under field condition because so many biotic and abiotic factors play role. Therefore, it is important to develop reliable techniques to screen for resistance to $H$. armigera under laboratory and/or field conditions.

The lines showing high antixenosis and stable resistance to $H$. armigera can be used in chickpea improvement programs. The resistance mechanisms like antibiosis, antixenosis and tolerance involved in resistant genotypes can be exploited in future breeding programs to develop varieties resistant to $H$. armigera in chickpea.

\section{References}

Armes N J, Bond G S and Cooker R J 1993 The laboratory culture and development of Helicoverpa armigera. Natural
Resources Institute, Chaltam U.K. Bulletin 57.

Blaney W M and Simmonds M S T 1990 The role of chemicals from legumes in mediating host selection by adults and larvae of Helicoverpa armigera: A behavioral and electropholic study (in F.) Host selection behaviour of Helicoverpa armigera. Summary Proceedings of the First Consultative Group Meeting, ICRISAT Center, Patancheru, Andhra Pradesh, India, 5-7 Mar 1990.

Clement S L El-Din, Sharaf E I D N, Weigand S and Lateef S S 1992 Screening techniques and sources of resistance to insect pests of cool season food legumes. (In En.) (Summaries in En.) Program and abstracts. 2nd International Food Legume Research Conference, Cairo, Egypt, 12-16 April 1992. Cairo, Egypt. International Food Legume Research Conference, Organizing Committee pp 22.

Clement S L El-Din, Sharaf E 1 D N, Weigand S and Lateef S S 1994 Research achievements in plant resistance to insect pests of cool season food legumes. Euphytica 73: 41-50.

Courtney S P and Kibota T 1990 Mother does not know the best: selection of hosts by ovipositing insects. In: Insect Plant Interactions (Ed. E A Bernays) 61-68.

Cowgill S E and Lateef S S 1996 Identification of antibiotic and antixenotic resistance to Helicoverpaarmigera (Lepidoptera: Noctuidae) in chickpea. Journal of Economic Entomology 89: 224-229.

Fitt G P 1986 The influence of shortage of hosts on the specificity of oviposition behaviour in species of Daucus (Diptera: Tephritidae). Physiological Entomology 11: 133-143. 
Hardwick D F 1965 The corn ear worm complex. Macmillan Society of Canada: $1-247$

King A B S 1994 Heliothis /Helicoverpa (Lepidoptera: Noctuidae) In Insect pests of cotton (eds Mathews, J P Tunstall), Wallyngford Oxon, U.K., CABI Pp 39106.

Lateef S S 1985 Gram pod borer, Heliothisarmigera (Hub.) resistance in chickpea. Agricultural Ecosystem and Environment 14: 95-102.

Minkenberg $\mathrm{O} \quad \mathrm{P} \quad \mathrm{J} \quad \mathrm{M}$, Tatar $\mathrm{M}$ and Rosenheim J A 1992 Egg load as a major source of variability in insect foraging and oviposition behaviour. Oikos 65: 134-142.Mustapha F A, Jallow A and Myron P Z 1998 Effects of egg load on the host selection behaviour of Helicoverpa armigera (hubner) (Lepidopetera: Noctuidae). Journal of the Australian Entomological Society 34: 71-73.

Painter R H 1951 Insect resistance in crop plants, McMillan, New York, USA $p_{p}$. 289.

Pearson E O 1 and Darling R C M 1958 The insect pests of cotton in tropical Africa. London, UK. Empire Cotton Growers and Common Wealth Institute of Entomology pp 355.

Prokopy R J, Roitberg R D, and Vargas R A 1994 Effects of egg load on finding and acceptance of host fruit in
Ceratitiscapitata.

Entomology 19: 124-132.

Singer M C 1982 Quantification of host preference by manipulation of oviposition behaviour in Euphydryaseditha. Oocologia52: 230235.

Sison M J, Cowgil E and Lateef S S 1996 Identification of antibiotic and antixenotic resistance to Helicoverpa armigera (Lepidoptera: Noctuidae) in chickpea. Journal of Economic Entomology 89: 224-228.

Sison M J, Shanower T G and Bhagwat V R 1993 Helicoverpa (Hubner) ovipositional and larval feeding preference among 6 short duration pigeonpea genotypes International Pigeonpea Newsletter, 17: 37-40.

Srivastava C P and Srivastava R P 1989 Screening for resistance to the gram pod borer $H$. armigera in chickpea genotypes and obviations on its mechanisms of resistance in India. Insect Science and its Application 10: 255-258.

Stevenson P C, Simmonds M S J, Green P W C and Sharma H C 2002 Physical and chemical mechanisms of plant resistance to Helicoverpa armigera: chickpea and pigeonpea - the way ahead (Sharma, H.C., eds.). Patancheru 502 324, Andhra Pradesh, India: International Crops Research Institute for the Semi-Arid Tropics

\section{How to cite this article:}

Sree Latha, E. and Sharma, H.C. 2018. Antixenosis Mechanism of Resistance to Helicoverpa armigera (Hub.) in Chickpea (Cicer arietinum Linn.). Int.J.Curr.Microbiol.App.Sci. 7(02): 1233-1238. doi: https://doi.org/10.20546/ijcmas.2018.702.151 